\title{
Gender differences in functional connectivities between insular subdivisions and selective pain-related brain structures
}

Yu-Jie Dai ${ }^{1,2,3^{\dagger}}$, Xin Zhang ${ }^{1 \dagger}$, Yang Yang ${ }^{1 \dagger}$, Hai-Yan Nan ${ }^{1}$, Ying Yu ${ }^{1}$, Qian Sun ${ }^{1}$, Lin-Feng Yan ${ }^{1}$, Bo Hu${ }^{1}$, Jin Zhang ${ }^{1}$, Zi-Yu Qiu ${ }^{4}$, Yi Gao ${ }^{4}$, Guang-Bin Cui ${ }^{1 *}$, Bi-Liang Chen ${ }^{2^{*}}$ and Wen Wang ${ }^{1 *}$ (D)

\begin{abstract}
Background: The incidence of pain disorders in women is higher than in men, making gender differences in pain a research focus. The human insular cortex is an important brain hub structure for pain processing and is divided into several subdivisions, serving different functions in pain perception. Here we aimed to examine the gender differences of the functional connectivities (FCs) between the twelve insular subdivisions and selected pain-related brain structures in healthy adults.
\end{abstract}

Methods: Twenty-six healthy males and 11 age-matched healthy females were recruited in this cross-sectional study. FCs between the 12 insular subdivisions (as 12 regions of interest (ROIs)) and the whole brain (ROl-whole brain level) or 64 selected pain-related brain regions (64 ROIs, ROI-ROI level) were measured between the males and females.

Results: Significant gender differences in the FCs of the insular subdivisions were revealed: (1) The FCs between the dorsal dysgranular insula ( $\mathrm{dld}$ ) and other brain regions were significantly increased in males using two different techniques (ROI-whole brain and ROI-ROI analyses); (2) Based on the ROI-whole brain analysis, the FC increases in 4 FC-pairs were observed in males, including the left dld - the right median cingulate and paracingulate/ right posterior cingulate gyrus/ right precuneus, the left dld - the right median cingulate and paracingulate, the left dld the left angular as well as the left dld - the left middle frontal gyrus; (3) According to the ROI-ROI analysis, increased FC between the left dld and the right rostral anterior cingulate cortex was investigated in males.

Conclusion: In summary, the gender differences in the FCs of the insular subdivisions with pain-related brain regions were revealed in the current study, offering neuroimaging evidence for gender differences in pain processing.

Trial registration: ClinicalTrials.gov, NCT02820974. Registered 28 June 2016.

Keywords: Gender differences, Insular subdivisions, Pain, Functional connectivity, Resting-state

\footnotetext{
*Correspondence: cgbtd@126.com; chenbiliang0728@126.com;

wangwen@fmmu.edu.cn

Yu-Jie Dai, Xin Zhang and Yang Yang contributed equally to this work.

†Equal contributors

'Department of Radiology \& Functional and Molecular Imaging Key Lab of

Shaanxi Province, Tangdu Hospital, the Military Medical University of PLA

Airforce (Fourth Military Medical University), 569 Xinsi Road, Xi'an, Shaanxi

Province 710038, China

2Department of Obstetrics and Gynecology, Xijing Hospital, the Military

Medical University of PLA Airforce (Fourth Military Medical University), 15

West Changle Road, Xi'an, Shaanxi Province 710032, China

Full list of author information is available at the end of the article
} 


\section{Background}

Pain is one of the most common complaints making people seek medical attention, affecting over one-quarter of the global population, and its incidence increases with aging [1]. Besides aging, gender is another important factor affecting pain procession due to its high prevalence in women [2]. In a large scale study, women report higher pain intensity scores than men under the similar pain conditions [3]. Besides, females are more frequently suffered from pain disorders, such as migraine [4, 5], temporomandibular joint disorder [6], fibromyalgia [7, 8] as well as irritable bowel syndrome (IBS) $[9,10]$. These findings suggest that there may be structural or functional gender differences in the brain matrix for pain processing in varied pain disorders [6]. Therefore, a thorough understanding of the central mechanisms underlying this gender differences in healthy people is pivotal for pain research.

Pain experience involves the interaction among sensory, emotional, cognitive, genetic and environmental factors [11] and pain processing is complicated, comprising a variety of brain regions working in concert. Functional magnetic resonance imaging (fMRI) technique has been performed to delineate a set of cortical and subcortical structures-the pain matrix-in pain perception [12, 13]. The classical pain matrix consists of three networks, i.e. the sensory, affective and cognitive networks [14-16]. The sensory network involves the lateral thalamus, primary (SI) and secondary (SII) somatic cortices, the insular cortex as well [17]. Among them, the insular cortex has been suggested to participate in both sensory-discriminative and affective-motivational aspects of pain processing [18]. The stronger pain activations of the somatosensory and the insular cortices in men while medial prefrontal cortex in women under pain challenging have been reported in the previous neuroimaging studies [19-21]. Furthermore, female IBS patients demonstrated significant functional alterations in the insular cortex than male patients [22]. Rather than evaluating evoked responses, it is possible to measure neural activity that is not linked to a specific stimulus or task. The most advanced approach is referred to as "functional connectivity (FC)" [23], which provides insight into how brain regions work together as network to produce pain and how these networks can become strengthened or weakened in pain disorders [24]. Due to the crucial role of insula in pain processing, its FCs with other pain-related brain regions may contribute to interpreting the above-mentioned gender differences. However, the insular cortex is functionally heterogeneous, consisting of multiple distinct subdivisions and the FCs between these subdivisions and other pain processing regions remain largely unknown.

In a recent study, insular cortex is divided into 6 subdivisions and the resting-state FCs between these 6 subdivisions and other 12 pain-related brain regions in both healthy men and women have been reported [25]. However, these subdivisions may not reflect the complexity of insular cortex. Recently, the insular cortex is divided into 12 subdivisions (http://atlas.brainnetome.org/) [26], but it has not been investigated the FCs between these 12 insular subdivisions and more painrelated brain structures, nor is it influenced by gender.

We thus designed the current study to compare the gender differences of FCs between 12 insular subdivisions and other pain-related brain regions by using 2 techniques: first, the gender differences of FCs between the 12 insular subdivisions and the whole brain (region of interest (ROI)-whole brain analysis); second, gender differences of FCs between the 12 insular subdivisions and the 64 selected pain-related brain regions (ROIROI analysis).

\section{Methods}

\section{Participants}

Thirty-seven healthy volunteers were recruited from the community participated in the trial, including 26 men (mean age, $49.46 \pm 3.75$ years) and 11 women (mean age, $52.09 \pm 5.65$ years). All subjects provided the written informed consent to the study prior to data collection. None participant possessed magnetic resonance imaging (MRI) examination contraindications. Exclusion criteria included neurological diseases or psychiatric disorders, severe internal disorders, concurrence of the cardiovascular system disease, pregnancy or lactation, and alcohol or tobacco dependence.

\section{Clinical characteristics analysis}

The statistical analysis was conducted by using of Statistical Program for Social Sciences (SPSS) 20.0 software. The demographic data of the two groups was compared using a two-sample $t$-test, and $p<0.05$ was considered as statistical significance.

\section{Resting-state functional data collection}

Resting-state blood oxygenation level dependent (BOLD) images were acquired on GE Discovery MR750 $3.0 \mathrm{~T}$ MR scanner with an eight-channel phased-array head coil at the Department of Radiology, Tangdu Hospital of the Fourth Military Medical University. Prior to scanning, the head of each subject was stabilized in order to minimize head motion. Moreover, the head position was monitored during the whole scanning. For each subject, structural T1 weighted imaging (T1WI) and T2 fluid attenuated inversion recovery (FLAIR) sequences were firstly employed to exclude apparent brain lesions. Resting-state BOLD images were collected utilizing an echo planar imaging (EPI) sequence. Through the scanning process, participants were placed in the supine position and informed to remain as motionless as 
possible, keep awake, relax their minds and think of nothing in particular. The EPI sequence scanning lasted for $6 \mathrm{~min}$ and $10 \mathrm{~s}$. The scanning parameters were as the following:

T1WI:flip angle $(\mathrm{FA})=111^{\circ}$, echo time $(\mathrm{TE})=24 \mathrm{~ms}$, number of echoes $=1$, repetition time $(\mathrm{TR})=1750 \mathrm{~ms}$, inversion time $(\mathrm{TI})=780 \mathrm{~ms}$, receiver bandwidth $=41.67$, field of view $(F O V)=24 \mathrm{~mm}^{2}$, slice thickness $=5 \mathrm{~mm}$, slice spacing $=1.5$, number of slices $=20$;

T2 FLAIR: $\mathrm{TE}=145 \mathrm{~ms}$, number of echoes $=1, \mathrm{TR}=$ $8400 \mathrm{~ms}, \mathrm{TI}=2100 \mathrm{~ms}$, receiver bandwidth $=83.33, \mathrm{FOV}=$ $24 \mathrm{~mm}^{2}$, slice thickness $=5 \mathrm{~mm}$, slice spacing $=1.5$, number of slices $=18$;

BOLD: $\mathrm{FA}=90^{\circ}, \mathrm{TE}=30 \mathrm{~ms}$, number of echoes $=1$, $\mathrm{TR}=2000 \mathrm{~ms}$, number of shots $=1, \mathrm{FOV}=22 \mathrm{~mm}^{2}$, slice thickness $=3 \mathrm{~mm}$, slice spacing $=1.0$, number of slices $=$ 36 , time points $=185$.

\section{Brain region masks}

\section{Masks for insular subdivisions}

According to the published Brainnetome Atlas (http:// atlas.brainnetome.org/download.html), the insular cortex is divided into 12 subdivisions, including the hypergranular (G), ventral agranular (vIa), dorsal agranular (dIa), ventral dysgranular and granular (vId/vIg), dorsal granular (dIg), dorsal dysgranular (dId) in left and right insular cortices [27]. The detailed information of the 12 insular subdivisions was presented in Table 1.

\section{Masks for selected pain-related brain regions}

Based on the neuroanatomical knowledge of brain as well as extensive literature reviewing, 64 pain-related brain regions were selected in the current fMRI study. The detailed information of these pain-related brain regions, including the location, label and Montreal Neurological Institute (MNI) coordinate, was shown in Table 2. Masks for most pain-related regions were selected from the Brainnetome Atlas, including ventral dorsolateral prefrontal cortex (vDLPFC), the opercular pars triangularis (oPT) and ventral pars triangularis (vPT), primary motor cortex (PMC), postcentral somatosensory association cortex (pSAC), the caudal supramarginal gyrus (CSG) and rostroventral supramarginal gyrus (rvSG), SI, the rostroventral ventral anterior cingulate cortex (rvVACC), caudal ventral anterior cingulate cortex (cvACC), pregenual dorsal anterior cingulate cortex (pdACC) and subgenual dorsal anterior cingulate cortex (sdACC), the dorsolateral putamen $(\mathrm{dlPu})$, the medial prefrontal thalamus (mPFtha), premotor thalamus (mPMtha), posterior parietal thalamus (PPtha), caudal temporal thalamus (cTtha), and lateral prefrontal thalamus (lPFtha), the medial amygdala (mAmyg) and lateral amygdala (lAmyg), the rostral hippocampus (rHipp) and caudal hippocampus (cHipp). Besides, the mask for SII was chosen from the Juelich Histological Atlas, which was distributed with FMRIB Software Library (FSL) tool. In addition, the specific MNI coordinates of the rest pain-related regions were referred to the published studies, including rostral anterior cingulate cortex (rACC), ventrolateral prefrontal cortex (VLPFC), posterior midcingulate cortex (pMCC), orbitofrontal cortex (OFC) as well as the ventrolateral periaqueductal gray (vlPAG), lateral periaqueductal gray (IPAG), and dorsolateral periaqueductal gray (dlPAG) [28].

Table $1 \mathrm{MNI}$ coordinates of the 12 insular subdivisions

\begin{tabular}{|c|c|c|c|c|c|c|}
\hline \multirow[t]{2}{*}{ Insular subdivision } & \multirow[t]{2}{*}{ Abbreviation } & \multirow{2}{*}{$\begin{array}{l}\text { Left and } \\
\text { right } \\
\text { hemisphere }\end{array}$} & \multirow[t]{2}{*}{ Label } & \multicolumn{3}{|c|}{ MNI coordinate } \\
\hline & & & & $\bar{x}$ & Y & Z \\
\hline Hypergranular insula & G & L & G_L & -36 & -20 & 10 \\
\hline Hypergranular insula & G & $\mathrm{R}$ & G_R & 37 & -18 & 8 \\
\hline Ventral agranular insula & vla & L & vla_L & -32 & 14 & -13 \\
\hline Ventral agranular insula & vla & $\mathrm{R}$ & vla_R & 33 & 14 & -13 \\
\hline Dorsal agranular insula & dla & L & dla_L & -34 & 18 & 1 \\
\hline Dorsal agranular insula & dla & $\mathrm{R}$ & dla_R & 36 & 18 & 1 \\
\hline Ventral dysgranular and granular insula & $\mathrm{vld} / \mathrm{vlg}$ & $L$ & vld/vlg_L & -38 & -4 & -9 \\
\hline Ventral dysgranular and granular insula & $\mathrm{vld} / \mathrm{vlg}$ & $\mathrm{R}$ & vld/vlg_R & 39 & -2 & -9 \\
\hline Dorsal granular insula & dlg & L & dlg_L & -38 & -8 & 8 \\
\hline Dorsal granular insula & $\mathrm{dlg}$ & $\mathrm{R}$ & dlg_R & 39 & -7 & 8 \\
\hline Dorsal dysgranular insula & dld & L & dld_L & -38 & 5 & 5 \\
\hline Dorsal dysgranular insula & dld & $\mathrm{R}$ & dld_R & 38 & 5 & 5 \\
\hline
\end{tabular}

$L$ left, $R$ right, $M N I$ Montreal Neurological Institute 
Table $2 \mathrm{MNI}$ coordinates of the 64 selected pain-related brain regions

\begin{tabular}{|c|c|c|c|c|c|c|c|}
\hline \multirow[t]{2}{*}{ Location } & \multirow[t]{2}{*}{ Brain region } & \multirow[t]{2}{*}{ Abbreviation } & \multirow{2}{*}{$\begin{array}{l}\text { Left and } \\
\text { right } \\
\text { hemisphere }\end{array}$} & \multirow[t]{2}{*}{ Label } & \multicolumn{3}{|c|}{ MNI coordinate } \\
\hline & & & & & $x$ & Y & Z \\
\hline Middle frontal gyrus & ventral dorsolateral prefrontal cortex & VDLPFC & $\mathrm{L}$ & VDLPFC_L & -41 & 41 & 16 \\
\hline Middle frontal gyrus & ventral dorsolateral prefrontal cortex & VDLPFC & $\mathrm{R}$ & VDLPFC_R & 42 & 44 & 14 \\
\hline Middle frontal gyrus & ventrolateral prefrontal cortex & VLPFC & L & VLPFC_L & -48 & 20 & -8 \\
\hline Middle frontal gyrus & ventrolateral prefrontal cortex & VLPFC & $\mathrm{R}$ & VLPFC_R & 48 & 20 & -8 \\
\hline Inferior frontal gyrus & opercular pars triangularis & oPT & L & OPT_L & -39 & 23 & 4 \\
\hline Inferior frontal gyrus & opercular pars triangularis & OPT & $\mathrm{R}$ & OPT_R & 42 & 22 & 3 \\
\hline Inferior frontal gyrus & ventral pars triangularis & VPT & L & VPT_L & -52 & 13 & 6 \\
\hline Inferior frontal gyrus & ventral pars triangularis & VPT & $\mathrm{R}$ & VPT_R & 54 & 14 & 11 \\
\hline Frontal gyrus & orbitofrontal cortex & OFC & $\mathrm{L}$ & OFC_L & -24 & 34 & -12 \\
\hline Frontal gyrus & orbitofrontal cortex & OFC & $\mathrm{R}$ & OFC_R & 24 & 34 & -12 \\
\hline Precentral gyrus & primary motor cortex & PMC & L & PMC_L & -52 & 0 & 8 \\
\hline Precentral gyrus & primary motor cortex & PMC & $\mathrm{R}$ & PMC_R & 54 & 4 & 9 \\
\hline Superior parietal lobe & postcentral somatosensory association cortex & pSAC & L & pSAC_L & -22 & -47 & 65 \\
\hline Superior parietal lobe & postcentral somatosensory association cortex & PSAC & $\mathrm{R}$ & pSAC_R & 23 & -43 & 67 \\
\hline Inferior parietal lobe & caudal supramarginal gyrus & CSG & $\mathrm{L}$ & CSG_L & -56 & -49 & 38 \\
\hline Inferior parietal lobe & caudal supramarginal gyrus & CSG & $\mathrm{R}$ & CSG_R & 57 & -44 & 38 \\
\hline Inferior parietal lobe & rostroventral supramarginal gyrus & rvSG & L & rvSG_L & -53 & -31 & 23 \\
\hline Inferior parietal lobe & rostroventral supramarginal gyrus & rvSG & $\mathrm{R}$ & rvSG_R & 55 & -26 & 26 \\
\hline Postcentral gyrus & $\begin{array}{l}\text { primary somatosensory cortex (tongue and } \\
\text { larvnx region) }\end{array}$ & $\mathrm{SI}$ & $\mathrm{L}$ & SI_L & -56 & -14 & 16 \\
\hline Postcentral gyrus & $\begin{array}{l}\text { primary somatosensory cortex (tongue and } \\
\text { larvnx region) }\end{array}$ & $\mathrm{SI}$ & $\mathrm{R}$ & SI_R & 56 & -10 & 15 \\
\hline Postcentral gyrus & primary somatosensory cortex (trunk region) & $\mathrm{SI}$ & L & SI_L & -21 & -35 & 68 \\
\hline Postcentral gyrus & primary somatosensory cortex (trunk region) & $\mathrm{SI}$ & $\mathrm{R}$ & SI_R & 20 & -33 & 69 \\
\hline Postcentral gyrus & secondary somatosensory cortex & SII & $L$ & SII_L & -52 & -26 & 22 \\
\hline Postcentral gyrus & secondary somatosensory cortex & SII & $\mathrm{R}$ & SII_R & 56 & -22 & 24 \\
\hline Cingulate gyrus & rostroventral ventral anterior cingulate cortex & rVVACC & $\mathrm{L}$ & rVVACC_L & -3 & 8 & 25 \\
\hline Cingulate gyrus & rostroventral ventral anterior cingulate cortex & rVVACC & $\mathrm{R}$ & rVVACC_R & 5 & 22 & 12 \\
\hline Cingulate gyrus & caudal ventral anterior cingulate cortex & CVACC & $\mathrm{L}$ & CVACC_L & -5 & 7 & 37 \\
\hline Cingulate gyrus & caudal ventral anterior cingulate cortex & CVACC & $\mathrm{R}$ & CVACC_R & 4 & 6 & 38 \\
\hline Cingulate gyrus & pregenual dorsal anterior cingulate cortex & pdACC & L & pdACC_L & -6 & 34 & 21 \\
\hline Cingulate gyrus & pregenual dorsal anterior cingulate cortex & pdACC & $\mathrm{R}$ & PdACC_R & 5 & 28 & 27 \\
\hline Cingulate gyrus & subgenual dorsal anterior cingulate cortex & sdACC & L & sdACC_L & -4 & 39 & -2 \\
\hline Cingulate gyrus & subgenual dorsal anterior cingulate cortex & sdACC & $\mathrm{R}$ & sdACC_R & 5 & 41 & 6 \\
\hline Cingulate gyrus & rostral anterior cingulate cortex & rACC & L & rACC_L & -7 & 27 & 29 \\
\hline Cingulate gyrus & rostral anterior cingulate cortex & rACC & $\mathrm{R}$ & rACC_R & 7 & 27 & 29 \\
\hline Cingulate gyrus & caudal ventral posterior cingulate cortex & cVPCC & L & CVPCC_L & -7 & -23 & 41 \\
\hline Cingulate gyrus & caudal ventral posterior cingulate cortex & CVPCC & $\mathrm{R}$ & CVPCC_R & 6 & -20 & 40 \\
\hline Cingulate gyrus & posterior midcingulate cortex & pMCC & L & pMCC_L & -3 & -21 & 51 \\
\hline Cingulate gyrus & posterior midcingulate cortex & $\mathrm{pMCC}$ & $\mathrm{R}$ & PMCC_R & 3 & -21 & 51 \\
\hline Basal ganglia & dorsolateral putamen & $\mathrm{dlPu}$ & $\mathrm{L}$ & $\mathrm{dlPu} L \mathrm{~L}$ & -28 & -5 & 2 \\
\hline Basal ganglia & dorsolateral putamen & $\mathrm{dlPu}$ & $\mathrm{R}$ & $d P u_{-} R$ & 29 & -3 & 1 \\
\hline Periaqueductal gray & ventrolateral periaqueductal gray & VIPAG & $\mathrm{L}$ & VIPAG_L & -3 & -32 & -12 \\
\hline
\end{tabular}


Table $2 \mathrm{MNI}$ coordinates of the 64 selected pain-related brain regions (Continued)

\begin{tabular}{|c|c|c|c|c|c|c|c|}
\hline \multirow[t]{2}{*}{ Location } & \multirow[t]{2}{*}{ Brain region } & \multirow[t]{2}{*}{ Abbreviation } & \multirow{2}{*}{$\begin{array}{l}\text { Left and } \\
\text { right } \\
\text { hemisphere }\end{array}$} & \multirow[t]{2}{*}{ Label } & \multicolumn{3}{|c|}{ MNI coordinate } \\
\hline & & & & & $x$ & Y & Z \\
\hline Periaqueductal gray & ventrolateral periaqueductal gray & VIPAG & $\mathrm{R}$ & VIPAG_R & 3 & -32 & -12 \\
\hline Periaqueductal gray & lateral periaqueductal gray & IPAG & L & IPAG_L & -4 & -31 & -8 \\
\hline Periaqueductal gray & lateral periaqueductal gray & IPAG & $\mathrm{R}$ & IPAG_R & 4 & -31 & -8 \\
\hline Periaqueductal gray & dorsolateral periaqueductal gray & dIPAG & $L$ & dIPAG_L & -2 & -32 & -5 \\
\hline Periaqueductal gray & dorsolateral periaqueductal gray & dIPAG & $\mathrm{R}$ & dIPAG_R & 2 & -32 & -5 \\
\hline Thalamus & medial prefrontal thalamus & mPFtha & L & mPFtha_L & -7 & -12 & 5 \\
\hline Thalamus & medial prefrontal thalamus & mPFtha & $\mathrm{R}$ & mPFtha_R & 7 & -11 & 6 \\
\hline Thalamus & premotor thalamus & mPMtha & $L$ & mPMtha_L & -18 & -13 & 3 \\
\hline Thalamus & premotor thalamus & mPMtha & $\mathrm{R}$ & mPMtha_R & 12 & -14 & 1 \\
\hline Thalamus & posterior parietal thalamus & PPtha & $L$ & PPtha_L & 16 & -24 & 6 \\
\hline Thalamus & posterior parietal thalamus & PPtha & $\mathrm{R}$ & PPtha_R & 15 & -25 & 6 \\
\hline Thalamus & caudal temporal thalamus & cTtha & $L$ & cTtha_L & -12 & -22 & 13 \\
\hline Thalamus & caudal temporal thalamus & cTtha & $\mathrm{R}$ & cTtha_R & 10 & -14 & 14 \\
\hline Thalamus & lateral prefrontal thalamus & IPFtha & $L$ & IPFtha_L & -11 & -14 & 2 \\
\hline Thalamus & lateral prefrontal thalamus & IPFtha & $\mathrm{R}$ & IPFtha_R & 13 & -16 & 7 \\
\hline Amygdala & medial amygdala & mAmyg & $L$ & mAmyg_L & -19 & -2 & -20 \\
\hline Amygdala & medial amygdala & mAmyg & $\mathrm{R}$ & mAmyg_R & 19 & -2 & -19 \\
\hline Amygdala & lateral amygdala & IAmyg & L & IAmyg_L & -27 & -4 & -20 \\
\hline Amygdala & lateral amygdala & IAmyg & $\mathrm{R}$ & IAmyg_R & 28 & -3 & -20 \\
\hline Hippocampus & rostral hippocampus & rHipp & L & rHipp_L & -22 & -14 & -19 \\
\hline Hippocampus & rostral hippocampus & rHipp & $\mathrm{R}$ & rHipp_R & 22 & -12 & -20 \\
\hline Hippocampus & caudal hippocampus & cHipp & L & CHipp_L & -28 & -30 & -10 \\
\hline Hippocampus & caudal hippocampus & cHipp & $\mathrm{R}$ & cHipp_R & 29 & -27 & -10 \\
\hline
\end{tabular}

$L$ left, $R$ right, $M N I$ Montreal Neurological Institute

\section{Processing of resting-state fMRI data Data preprocessing}

The preprocessing of the BOLD images was performed by using the Data Processing Assistant for Resting-State fMRI (DPARSF, Yan and Zang 2010, http://rfmri.org/ DPARSF), which is based on Statistical Parametric Mapping (SPM8, http://www.fil.ion.ucl.ac.uk/spm) and the toolbox for Data Processing \& Analysis of Brain Imaging (DPABI, Yan et al. 2016, http://rfmri.org/DPABI). Briefly, the first 10 volumes of each subject were discarded in order to reach the signal equilibration and allow the subjects to adapt to the scanning environment. The remained scans were corrected for acquisition time differences between different slices and next realigned to the middle time point to correct for head motion. Then the head motion parameters of the subject were obtained and assessed with a maximum rotation less than $1^{\circ}$ or a maximum displacement less than $1 \mathrm{~mm}$. Nuisance regression was applied on white matter and cerebrospinal fluid, separately. Then the motion-corrected BOLD images were spatially normalized by using EPI templates and resampled with a voxel size of
$3 \times 3 \times 3 \mathrm{~mm}^{3}$. After spatial normalization, the images were spatially smoothed with a Gaussian kernel of $4 \mathrm{~mm}$ fullwidth at half maximum (FWHM) to reduce spatial noise. In the end, a band-pass filter $(0.01 \mathrm{~Hz}<\mathrm{f}<0.08 \mathrm{~Hz})$ was utilized to remove the effects of low frequency physiological drift and high frequency noise. After performing these steps, a 4-dimensional residual time series dataset was set up for each subject in the standard MNI space.

\section{FCs analysis between the 12 insular subdivisions and the whole brain}

The ROI-whole brain analysis was conducted between the 12 insular subdivisions (a total of 12 ROIs) and the whole brain. The mean time series for each ROI were calculated and then correlated with the time courses of all other voxels in the brain for each subject. Pearson correlation coefficients were converted to normally distributed scores by use of the Fisher's r-to-z transformation [23]. Group-level analysis for the general linear model was performed applying two sample $t$-test between the z-scores of the male and female groups. 
The reported results of the ROI-whole brain correlation analysis were carried out performing an uncorrected peak level of $p<0.001$ to correct for false positive rates and a false discovery rate (FDR) correction by using SPM8 at cluster level for multiple comparisons with threshold of $p<0.05[29,30]$.

\section{FCs analysis between the 12 insular subdivisions and the 64 selected pain-related regions}

Next, 64 brain regions that were recognized to be associated with pain were selected to calculate the ROI-ROI level FCs with the 12 sub-insular divisions (a total of 12 ROIs). Similar to the above-mentioned ROI-whole brain level analysis, Fisher's r-to-z transformation was conducted to increase the normality of the fMRI data. Based on the $12 \times 76 \mathrm{z}$-FC matrix, two-sample $t$-test was performed between the male group and the female group. FDR correction with threshold of $p<0.05$ for multiple comparison was then employed using MATLAB 2012b and to identify the final FC pairs with significant difference attributed to the gender factor.

\section{Results}

\section{Demographic data}

The demographic data of the two groups were shown in Table 3. No significant differences were revealed between male and female groups in age, hand dominance as well as educational level.

\section{Gender differences in ROI-whole brain level FCs}

FCs between the 12 insular subdivisions and whole brain in male and female groups were first investigated. Male subjects showed significantly increased FCs between the left dId and other four brain regions, comprising the voxels containing the right median cingulate and paracingulate/ right posterior cingulate gyrus/ right precuneus, the right median cingulate and paracingulate, the left angular, and the left middle frontal gyrus (Table 4 and Fig. 1a). The surface visualization of these significantly increased clusters in males was presented in Fig. 1b.

Table 3 Demographic characteristics

\begin{tabular}{llll}
\hline & $\begin{array}{l}\text { Male group } \\
(n=26)\end{array}$ & $\begin{array}{l}\text { Female group } \\
(n=11)\end{array}$ & $P$ \\
\hline Age (years) & $49.46 \pm 3.75$ & $52.09 \pm 5.65$ & 0.104 \\
Hand dominance (L/R) & $2 / 24$ & $1 / 10$ & 1.000 \\
Education (years) & $13.54 \pm 3.27$ & $12.18 \pm 3.22$ & 0.259 \\
\hline
\end{tabular}

Continuous data were expressed as mean \pm standard deviation (SD) $L$ left, $R$ right

\section{Gender differences in ROI-ROI level FCs}

FCs between the insular subdivisions and selected painrelated brain regions in male and female groups were compared using ROI-ROI level analysis. In line with the above ROI-whole brain analysis, the significant gender differences of FCs were also investigated between the 12 insular subdivisions and the 64 selected pain-related brain regions. Only the FC between the left dId and the right $\mathrm{rACC}$ in the total $12 \times 76 \mathrm{z}$-FC matrix was increased in the male subjects. The gender differences of 1 FC-pair with significance between the insular subdivisions and pain-related brain regions were shown in Fig. 2.

After merging the results of ROI-whole brain and ROI-ROI analyses, the dId in the left insular cortex was the primary insular subdivision that showed significantly increased FCs with the brain structures predominantly located in the right cingulate cortex in male subjects, shown in Fig. 3.

\section{Discussion}

In the current study, two analytical approaches were performed to explore the gender differences of FCs in insular subdivisions. Both ROI-whole brain and ROIROI analyses suggested that the FCs between left dId and right cingulate cortex were significantly increased in males. Our findings suggested that the increased FCs between left dId - right cingulate cortex in males played inhibiting role in the transmission and modulation of pain signals, thus decreased the pain intensity and incidence in male population.

According to the cytoarchitecture of the insular cortex, the dId locates in the posterior area of insula and participates in the sensory components of pain perception [27, 31]. In migraine patients, the dId was negatively connected to the median cingulate and paracingulate [25]. Female migraine patients also exhibited significantly decreased FCs between the posterior insula and the posterior cingulate cortex [5]. In addition to the above-mentioned gender differences in the clinical experience of migraine pain, healthy males in the current study showed statistically increased FCs between the insular subdivision and the cingulate cortex. Taking together the previous studies and our current one, the FCs between the insular and cingulate cortices were different between males and females, suggesting the functional distinctions in the brain matrix for pain processing among male and female populations.

Previous fMRI studies on gender differences in healthy subjects suggested that the male insular cortex is more activated under similar pain stimuli [19], however, without details of the activated subdivisions. Our study offered evidence that the male dorsal dysgranular area of the posterior insula was more spontaneously 
Table 4 Significant FCs between the insular subdivision and the whole brain in males

\begin{tabular}{|c|c|c|c|c|c|c|c|c|}
\hline \multirow{2}{*}{$\begin{array}{l}\text { Insular } \\
\text { subdivision }\end{array}$} & \multirow{2}{*}{$\begin{array}{l}\text { Cluster } \\
\text { index }\end{array}$} & \multirow[t]{2}{*}{ Brain region } & \multirow{2}{*}{$\begin{array}{l}\text { Cluster } \\
\text { size }\end{array}$} & \multicolumn{3}{|c|}{ MNI coordinate } & \multirow{2}{*}{$\begin{array}{l}\text { Peak } \\
\text { T-value }\end{array}$} & \multirow{2}{*}{$\begin{array}{l}\text { FCs } \\
\text { increased/ } \\
\text { decreased }\end{array}$} \\
\hline & & & & $x$ & Y & Z & & \\
\hline \multirow[t]{4}{*}{$\overline{d l d \_L}$} & 1 & $\begin{array}{l}\text { Cingulum_Mid/Cingulum_ } \\
\text { Post/Precuneus_R }\end{array}$ & 73 & 6 & -45 & 39 & 4.82 & Increased \\
\hline & 2 & Cingulum_Mid_R & 144 & 6 & 9 & 36 & 4.76 & Increased \\
\hline & 3 & Angular_L & 71 & -39 & -72 & 48 & 4.96 & Increased \\
\hline & 4 & Frontal_Mid_L & 56 & -42 & 21 & 39 & 4.53 & Increased \\
\hline
\end{tabular}

The threshold was set at uncorrected peak level of $p<0.001$, FDR correction with threshold of $p<0.05$, cluster size $\geq 50$ voxels. T-values of significantly activated peak-voxels referred to MNI coordinates. Brain region labeling was performed using the AAL atlas. T statistics and MNI coordinates were reported for the peak voxel within each cluster. AAL, Automatic Anatomic Labeling; FCs, functional connectivities; L, left; R, right; MNI, Montreal Neurological Institute; dld, dorsal dysgranular insula; Cingulum_Mid, median cingulate and paracingulate gyrus; Cingulum_Post, posterior cingulate gyrus; Frontal_Mid, middle frontal gyrus

activated than females. Since the posterior insula primarily plays an important role in the sensory network of pain [32, 33], our data suggested the potential involvement of dorsal dysgranular area of the posterior insula in the different pain processing in males and females under pain disorders. Reduced activity in the posterior insula may contribute to increased pain thresholds [34], suggesting its role in inhibiting pain response. However, the detailed underlying mechanisms need to be further investigated.
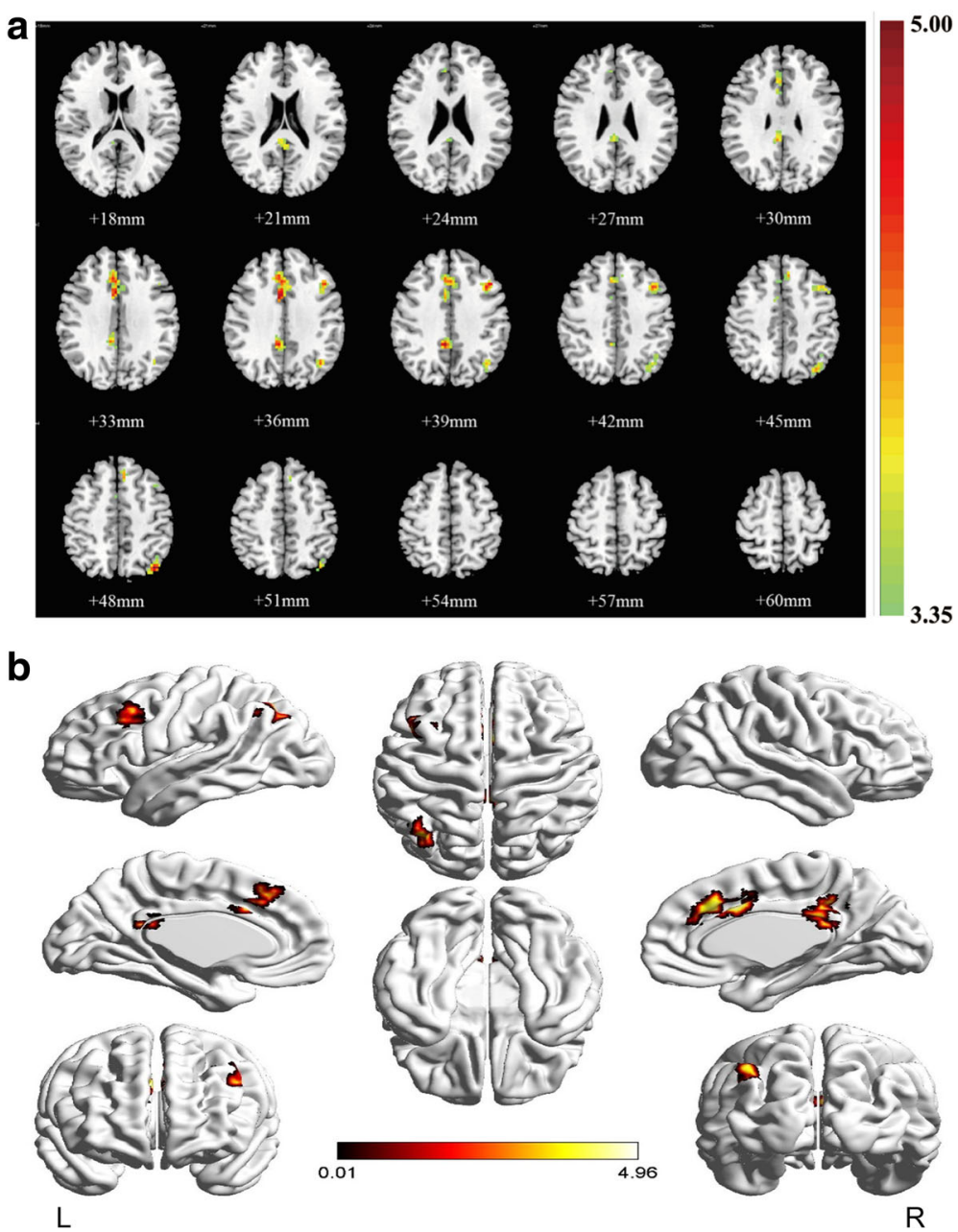

Fig. 1 ROI-whole brain analysis of FCs between the 12 insular subdivisions and the whole brain (visualization of the clusters on the brain surface). The warm color in the statistical differences map indicated the increased FCs between the dld_L and the whole brain in males. dld, dorsal dysgranular insula; L, left; R, right; FCs, functional connectivities; ROI, region of interest 


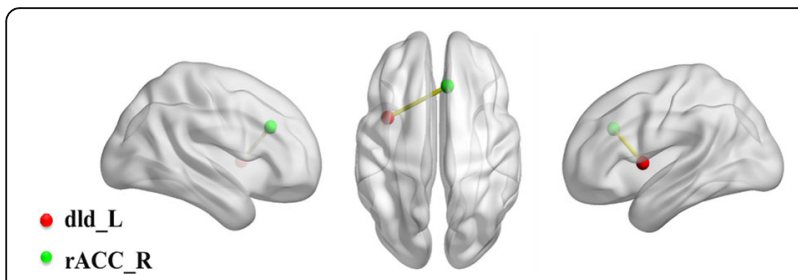

Fig. 2 ROI-ROI analysis of FCs between the 12 insular subdivisions and 64 selected pain-related brain regions. The red ball represented the dld_L, the green ball represented the rACC_R, and the yellow rod represented the statistically increased FC in males. L, left; R, right; dld, dorsal dysgranular insula; rACC, rostral anterior cingulate cortex; $\mathrm{ROI}$, region of interest; $\mathrm{FCs}$, functional connectivities

To our knowledge, information exchange between the insular and cingulate cortices has never been directly revealed. However, tracing studies in the monkey demonstrated that the posterior insula was connected to Brodmann areas 23 and 24 of the cingulate cortex [35], supporting our current findings. Based on the non-human primate functional neuroimaging studies, a co-activation of the insular and cingulate cortices was revealed in varied tasks [32, 36]. Furthermore, the posterior insular and the cingulate cortices were

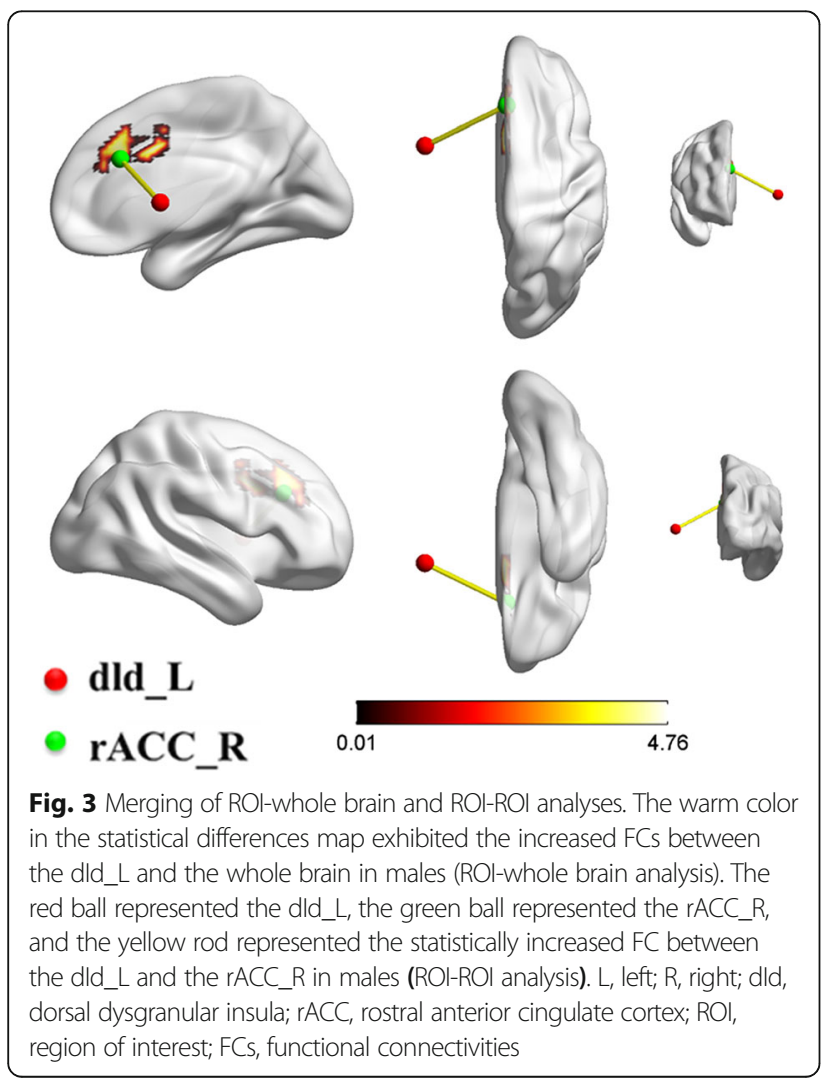

activated simultaneously in fibromyalgia patients [37]. Therefore, the distinct anatomical connections identified in the monkey combined with the evidence from functional imaging studies showing co-activation within specific areas of the insula and cingulate cortex, indicating multiple information processing pathways between the two brain structures [36]. Interestingly, the co-activation of the insular subdivision and the cingulate cortex also showed the gender differences in the current study. The FCs between the dId and cingulate cortex were significantly increased in male subjects. The FCs between insular subdivisions and other painrelated brain regions were also investigated in a recent study [25]. Despite the similar study design, there were apparent differences between their findings and ours. First, Wiech explored FCs between the insular subdivisions and other pain-related regions using the ROI-ROI method [25]. While we aimed to investigate that how the FCs between the insular subdivisions and other pain-relevant regions were influenced by gender difference (ROI-ROI analysis). In addition, we also identified the gender differences in FCs between the insular subdivisions and the whole brain (ROI-whole brain analysis). Second, the insular cortex was divided into 6 subdivisions in the previous study, while 12 subdivisions were utilized in the current study. Third, 12 painrelated brain regions were selected in Wiech's study, while 64 pain-related regions were selected in the present study based on an existing neuroanatomical knowledge and extensive literature review. Wiech revealed strongest FCs between the posterior insula and SII in 36 healthy adults (21 men and 15 women). Our study offered further information that the dId of the posterior insula is a major hub to cingulate cortex, performing pain inhibiting role.

There was limitation for our study. No pain-related psychological questionnaires were collected in the current study. Based on the existing evidence, healthy subjects also possess the pain-relevant psychological characteristics, such as pain vigilance and pain awareness [25]. The correlation analysis might reveal the potential relation between the FCs and the psychological traits of pain.

\section{Conclusion}

In conclusion, the present study identified the genderrelevant alterations in FCs of the insular subdivisions with other pain-related brain regions using two different methods. Specifically, men seem to have more access to a dId-mediated recruitment of the pain inhibition system than women. Given this, it is crucial for future study to take gender into account when probing the basic mechanisms of pain processing. 


\section{Abbreviations}

AAL: Automatic anatomic labeling; BOLD: Blood oxygenation level dependent; DPABI: Data processing \& analysis of brain imaging; DPARSF: Data processing assistant for resting-state fMRl; EPI: Echo planar imaging; FA: Flip angle; FC: Functional connectivity; FLAIR: Fluid attenuated inversion recovery; fMRI: Functional magnetic resonance imaging; FOV: Field of view; FSL: FMRIB software library; FWHM: Full-width at half maximum; IBS: IRRITABLE bowel syndrome; L: Left; MNI: Montreal neurological Institute; MRI: Magnetic resonance imaging; R: Right; ROl: Regions of interest; SD: Standard deviation; SI: Primary somatic cortices; SII: Secondary somatic cortices; SPM: Statistical parametric mapping; SPSS: Statistical program for social sciences; T1WI: T1 weighted imaging; TE: Echo time; TI: Inversion time; TR: Repetition time; VLPFC: Ventrolateral prefrontal cortex

\section{Funding}

This paper was sponsored by the National Natural Science Foundation of China (No. 81571656 and No. 61603399), the National Key Research and Development Program of China (No. 2016YFC0107105), the Innovation Foundation of Tangdu Hospital (No. 2016LCYJ011), and Key Project for Science Research and Development of Shaanxi Province (2017SF-012).

\section{Availability of data and materials}

All relevant data are within the paper.

\section{Authors' contributions}

DYJ, ZX, YY and WW contributed to all stages, including data acquisition and analysis, drafting the manuscript and revising for important intellectual content, as well as final approval of the version to be submitted. WW, NHY, $C G B$, and CBL were integral to the conception and design of the study. YY, $Y L F, H B$ and $Z J$ were responsible to the analysis and interpretation of the imaging data. SQ, QZY, and GY assisted with data collection in addition to the drafting and revising of this article. All authors read and approved the final manuscript.

\section{Ethics approval and consent to participate}

This study conformed to the principles of the Declaration of Helsinki and was approved by the ethics committee of Tangdu Hospital of the Fourth Military Medical University.

\section{Consent for publication}

Not applicable.

\section{Competing interests}

The authors declare that they have no competing interests.

\section{Publisher's Note}

Springer Nature remains neutral with regard to jurisdictional claims in published maps and institutional affiliations.

\begin{abstract}
Author details
${ }^{1}$ Department of Radiology \& Functional and Molecular Imaging Key Lab of Shaanxi Province, Tangdu Hospital, the Military Medical University of PLA Airforce (Fourth Military Medical University), 569 Xinsi Road, Xi'an, Shaanxi Province 710038, China. ${ }^{2}$ Department of Obstetrics and Gynecology, Xijing Hospital, the Military Medical University of PLA Airforce (Fourth Military Medical University), 15 West Changle Road, Xi'an, Shaanxi Province 710032 China. ${ }^{3}$ Department of Clinical Nutrition, Xijing Hospital, the Military Medica University of PLA Airforce (Fourth Military Medical University), 15 West Changle Road, Xi'an, Shaanxi Province 710032, China. ${ }^{4}$ Student Brigade, the Military Medical University of PLA Airforce (Fourth Military Medical University), 169 West Changle Road, Xi'an, Shaanxi Province 710032, China.
\end{abstract}

Received: 28 January 2018 Accepted: 24 February 2018

Published online: 14 March 2018

\section{References}

1. Mogil JS (2012) Sex differences in pain and pain inhibition: multiple explanations of a controversial phenomenon. Nat Rev Neurosci 13(12):859866. https://doi.org/10.1038/nrn3360
2. Fillingim RB, King CD, Ribeiro-Dasilva MC, Rahim-Williams B, Riley J 3rd (2009) Sex, gender, and pain: a review of recent clinical and experimental findings. J Pain 10(5):447-485. https://doi.org/10.1016/j.jpain.2008.12.001

3. Ruau D, Liu LY, Clark JD, Angst MS, Butte AJ (2012) Sex differences in reported pain across 11,000 patients captured in electronic medical records. J Pain 13(3):228-234. https://doi.org/10.1016/j.jpain.2011.11.002

4. Le H, Tfelt-Hansen P, Russell MB, Skytthe A, Kyvik KO, Olesen J (2011) Co-morbidity of migraine with somatic disease in a large population-based study. Cephalalgia 31(1):43-64. https://doi.org/10.1177/0333102410373159

5. Maleki N, Linnman C, Brawn J, Burstein R, Becerra L, Borsook D (2012) Her versus his migraine: multiple sex differences in brain function and structure. Brain 135(Pt 8):2546-2559. https://doi.org/10.1093/brain/aws175

6. Linnman C, Beucke JC, Jensen KB, Gollub RL, Kong J (2012) Sex similarities and differences in pain-related periaqueductal gray connectivity. Pain 153(2):444-454. https://doi.org/10.1016/j.pain.2011.11.006

7. Napadow V, Kim J, Clauw DJ, Harris RE (2012) Decreased intrinsic brain connectivity is associated with reduced clinical pain in fibromyalgia. Arthritis Rheum 64(7):2398-2403. https://doi.org/10.1002/art.34412

8. Napadow V, LaCount L, Park K, As-Sanie S, Clauw DJ, Harris RE (2010) Intrinsic brain connectivity in fibromyalgia is associated with chronic pain intensity. Arthritis Rheum 62(8):2545-2555. https://doi.org/10.1002/art.27497

9. Hong JY, Kilpatrick LA, Labus JS, Gupta A, Katibian D, Ashe-McNalley C, Stains J, Heendeniya N, Smith SR, Tillisch K, Naliboff B, Mayer EA (2014) Sex and disease-related alterations of anterior insula functional connectivity in chronic abdominal pain. J Neurosci 34(43):14252-14259. https://doi.org/10. 1523/JNEUROSCI.1683-14.2014

10. Gupta A, Kilpatrick L, Labus J, Tillisch K, Braun A, Hong JY, Ashe-McNalley C, Naliboff B, Mayer EA (2014) Early adverse life events and resting state neural networks in patients with chronic abdominal pain: evidence for sex differences. Psychosom Med 76(6):404-412. https://doi.org/10.1097/PSY. 0000000000000089

11. Rainville $P$ (2002) Brain mechanisms of pain affect and pain modulation. Curr Opin Neurobiol 12(2):195-204

12. Bastuji H, Frot M, Perchet C, Magnin M, Garcia-Larrea L (2016) Pain networks from the inside: spatiotemporal analysis of brain responses leading from nociception to conscious perception. Hum Brain Mapp 37(12):4301-4315. https://doi.org/10.1002/hbm.23310

13. Garcia-Larrea L, Peyron R (2013) Pain matrices and neuropathic pain matrices: a review. Pain 154(Suppl 1):S29-S43. https://doi.org/10.1016/j.pain. 2013.09.001

14. Apkarian AV, Bushnell MC, Treede RD, Zubieta JK (2005) Human brain mechanisms of pain perception and regulation in health and disease. Eur J Pain 9(4):463-484. https://doi.org/10.1016/j.ejpain.2004.11.001

15. Hofbauer RK, Rainville P, Duncan GH, Bushnell MC (2001) Cortical representation of the sensory dimension of pain. J Neurophysiol 86(1):402411. https://doi.org/10.1152/jn.2001.86.1.402

16. Tracey I, Mantyh PW (2007) The cerebral signature for pain perception and its modulation. Neuron 55(3):377-391. https://doi.org/10.1016/j. neuron.2007.07.012

17. A CNC (2008) Pain perception and its genesis in the human brain. Sheng Li Xue Bao 60(5):677-685

18. Monroe TB, Fillingim RB, Bruehl SP, Rogers BP, Dietrich MS, Gore JC, Atalla SW, Cowan RL (2017) Sex differences in brain regions modulating pain among older adults: a cross-sectional resting state functional connectivity study. Pain Med. https://doi.org/10.1093/pm/pnx084

19. Kong J, Loggia ML, Zyloney C, Tu P, Laviolette P, Gollub RL (2010) Exploring the brain in pain: activations, deactivations and their relation. Pain 148(2): 257-267. https://doi.org/10.1016/j.pain.2009.11.008

20. Moulton EA, Keaser ML, Gullapalli RP, Maitra R, Greenspan JD (2006) Sex differences in the cerebral BOLD signal response to painful heat stimuli. Am J Physiol Regul, Integrat Comp Physiol 291(2):R257-R267. https://doi.org/10. 1152/ajpregu.00084.2006

21. Straube T, Schmidt S, Weiss T, Mentzel HJ, Miltner WH (2009) Sex differences in brain activation to anticipated and experienced pain in the medial prefrontal cortex. Hum Brain Mapp 30(2):689-698. https://doi.org/10.1002/ hbm.20536

22. Hong JY, Kilpatrick LA, Labus J, Gupta A, Jiang Z, Ashe-McNalley C, Stains J, Heendeniya N, Ebrat B, Smith S, Tillisch K, Naliboff B, Mayer EA (2013) Patients with chronic visceral pain show sex-related alterations in intrinsic oscillations of the resting brain. J Neurosci 33(29):11994-12002. https://doi. org/10.1523/JNEUROSCI.5733-12.2013 
23. Liu F, Wang Y, Li M, Wang W, Li R, Zhang Z, Lu G, Chen H (2017) Dynamic functional network connectivity in idiopathic generalized epilepsy with generalized tonic-clonic seizure. Hum Brain Mapp 38(2):957-973. https://doi. org/10.1002/hbm.23430

24. Davis KD, Moayedi M (2013) Central mechanisms of pain revealed through functional and structural MRI. J Neurolmmune Pharmacol 8(3):518-534. https://doi.org/10.1007/s11481-012-9386-8

25. Wiech K, Jbabdi S, Lin CS, Andersson J, Tracey I (2014) Differential structural and resting state connectivity between insular subdivisions and other painrelated brain regions. Pain 155(10):2047-2055. https://doi.org/10.1016/j.pain. 2014.07.009

26. Fan L, Li H, Zhuo J, Zhang Y, Wang J, Chen L, Yang Z, Chu C, Xie S, Laird AR, Fox PT, Eickhoff SB, Yu C, Jiang T (2016) The human Brainnetome atlas: a new brain atlas based on connectional architecture. Cereb Cortex 26(8): 3508-3526. https://doi.org/10.1093/cercor/bhw157

27. Yu ZB, Lv YB, Song LH, Liu DH, Huang XL, Hu XY, Zuo ZW, Wang Y, Yang $Q$, Peng J, Zhou ZH, Li HT (2017) Functional connectivity differences in the insular sub-regions in migraine without Aura: a resting-state functional magnetic resonance imaging study. Front Behav Neurosci 11:124. https:// doi.org/10.3389/fnbeh.2017.00124

28. Coulombe MA, Erpelding N, Kucyi A, Davis KD (2016) Intrinsic functional connectivity of periaqueductal gray subregions in humans. Hum Brain Mapp 37(4):1514-1530. https://doi.org/10.1002/hbm.23117

29. Chumbley J, Worsley K, Flandin G, Friston K (2010) Topological FDR for neuroimaging. Neuro Image 49(4):3057-3064. https://doi.org/10.1016/j. neuroimage.2009.10.090

30. Liu F, Zhu C, Wang Y, Guo W, Li M, Wang W, Long Z, Meng Y, Cui Q, Zeng L, Gong Q, Zhang W, Chen H (2015) Disrupted cortical hubs in functional brain networks in social anxiety disorder. Clin Neurophysiol 126(9):17111716. https://doi.org/10.1016/j.clinph.2014.11.014

31. Kurth F, Zilles K, Fox PT, Laird AR, Eickhoff SB (2010) A link between the systems: functional differentiation and integration within the human insula revealed by meta-analysis. Brain Struct Funct 214(5-6):519-534. https://doi. org/10.1007/s00429-010-0255-z

32. Rance M, Ruttorf M, Nees F, Schad LR, Flor H (2014) Real time fMRI feedback of the anterior cingulate and posterior insular cortex in the processing of pain. Hum Brain Mapp 35(12):5784-5798. https://doi.org/10.1002/hbm.22585

33. Frot M, Magnin M, Mauguiere F, Garcia-Larrea L (2007) Human SII and posterior insula differently encode thermal laser stimuli. Cereb Cortex 17(3): 610-620. https://doi.org/10.1093/cercor/bhk007

34. Bar KJ, Berger S, Schwier C, Wutzler U, Beissner F (2013) Insular dysfunction and descending pain inhibition in anorexia nervosa. Acta Psychiatr Scand 127(4):269-278. https://doi.org/10.1111/j.1600-0447.2012.01896.x

35. Mesulam MM, Mufson EJ (1982) Insula of the old world monkey. III: efferent cortical output and comments on function. J Comp Neurol 212(1):38-52. https://doi.org/10.1002/cne.902120104

36. Taylor KS, Seminowicz DA, Davis KD (2009) Two systems of resting state connectivity between the insula and cingulate cortex. Hum Brain Mapp 30(9):2731-2745. https://doi.org/10.1002/hbm.20705

37. Ichesco E, Schmidt-Wilcke T, Bhavsar R, Clauw DJ, Peltier SJ, Kim J, Napadow V, Hampson JP, Kairys AE, Williams DA, Harris RE (2014) Altered resting state connectivity of the insular cortex in individuals with fibromyalgia. J Pain 15(8):815-826 e811. https://doi.org/10.1016/j.jpain.2014.04.007

\section{Submit your manuscript to a SpringerOpen ${ }^{\circ}$ journal and benefit from:}

- Convenient online submission

- Rigorous peer review

- Open access: articles freely available online

- High visibility within the field

- Retaining the copyright to your article

Submit your next manuscript at $\gg$ springeropen.com 